\title{
L'accompagnement d'enseignants d'éducation physique et à la santé dans la mise en place de stratégies visant à optimiser leurs pratiques évaluatives en éducation à la santé
}

\section{HRONIQUE • Recherche étudiante}

\section{Introduction}

L'éducation à la santé (ÉS) vise à responsabiliser les élèves vis-à-vis de leur propre santé. Il est toutefois difficile de trouver le meilleur emplacement pour l'ÉS à l'école. Au Québec, elle est intégrée dans le programme de formation du primaire de deux manières, soit : a) dans le domaine général de formation Santé et bien-être, b) en éducation physique et à la santé (ÉPS) à travers la compétence disciplinaire Adopter un mode de vie sain et actif (Grenier, 2009). Cette inclusion de l'ÉS en ÉPS modifie le rôle des enseignants de cette discipline et représente un défi de taille pour ces derniers (Turcotte, 2010).

\section{Problématique}

Dans leur recherche par questionnaire effectuée auprès de 223 enseignants d'ÉPS, Turcotte et al. (2011) identifient l'évaluation des apprentissages comme l'un des deux principaux besoins de formation continue en ÉS. Les formations continues en ÉS sont toutefois quasi inexistantes et ne permettent donc pas de répondre aux besoins de formation des enseignants d'ÉPS en matière d'évaluation (Alfrey, Webb et Cale, 2012). L'enjeu lié à l'inclusion de l'ÉS en ÉPS est donc la mise en place de formations continues qui favorisent le développement professionnel (DP) des enseignants d'ÉPS, et du même coup, une optimisation de leurs pratiques évaluatives en ÉS. Le dispositif de formation que l'on continue à privilégier semble être l'accompagnement professionnel, puisqu'un tel dispositif respecte plusieurs principes de DP identifiés dans la littérature scientifique, 
notamment ceux de soutenir les enseignants dans leurs réflexions individuelles et collectives (Hunzicker, 2010) et de faire évoluer leurs pratiques enseignantes (Lafortune, Lepage, Persechino et Bélanger, 2008). Ce projet de recherche doctorale vise ainsi à répondre à la question suivante : en quoi la mise en place d'un dispositif d'accompagnement professionnel module-t-elle les pratiques évaluatives en ÉS d'enseignants d'ÉPS du primaire?

\section{Cadre conceptuel}

La littérature scientifique offre très peu de modèles opérationnels qui sont spécifiques à l'accompagnement professionnel. Dans le cadre de la présente recherche, un modèle intégrateur a donc été créé en s'inspirant des étapes proposées dans deux modèles de recherche-action ( $\mathrm{McNiff}$ et Whitehead, 2011; Stringer, 2007) tout en y ajoutant certaines particularités de l'accompagnement professionnel (Biémar, 2012; Lafortune et al., 2008).

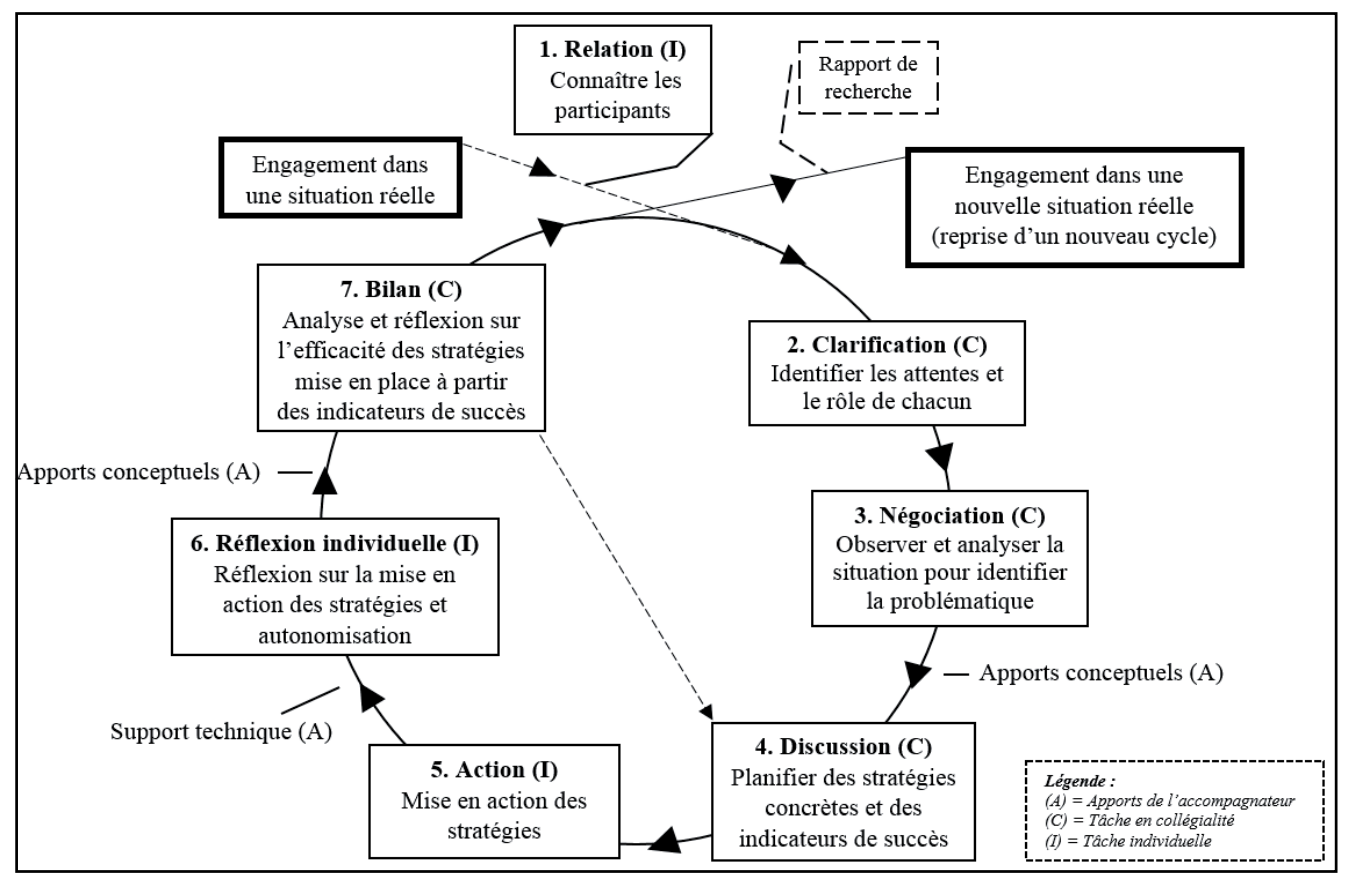

\section{Figure 1}

Modèle intégrateur en accompagnement professionnel. 
Lors d'un premier cycle d'accompagnement, l'accompagnateur s'entretient avec chacun des participants afin de connaître leur représentation de l'évaluation en ÉS et leurs besoins individuels (étape de relation). Par la suite, la démarche d'accompagnement s'opérationnalise à travers six autres étapes : 1) la clarification, où les attentes et le rôle de chacun sont identifiés; 2) la négociation, où une problématique collective est identifiée; 3) la discussion, où des stratégies concrètes et des indicateurs de succès sont planifiés; 4) l'action, où les stratégies planifiées sont mises en place; 5) la réflexion individuelle, où l'autonomisation de l'enseignant est favorisée grâce à une analyse réflexive sur la mise en action des stratégies et 6) l'étape de bilan, où l'efficacité des stratégies mises en place est analysée à partir des indicateurs de succès. Ensuite, les participants décident s'ils s'engagent dans une nouvelle problématique ou s'ils retournent à l'étape de discussion afin de trouver de nouvelles stratégies.

Les objectifs de la présente recherche sont de décrire l'opérationnalisation des stratégies élaborées par les enseignants d'ÉPS par rapport à leurs pratiques évaluatives en ÉS, de décrire le dispositif d'accompagnement professionnel mis en place avec les participants, et de décrire le processus d'autonomisation des enseignants d'ÉPS au regard de leurs pratiques évaluatives en ÉS.

\section{Méthodologie}

La recherche-action-formation s'avère une stratégie de recherche appropriée afin d'accompagner trois enseignants d'ÉPS du primaire ayant un intérêt particulier pour l'évaluation en ÉS. Ce type de recherche permet en effet de contribuer au questionnement des pratiques et d'amener les personnes concernées à effectuer une transformation de ces dernières (Guay et Prud'Homme, 2011). Les méthodes de collecte de données utilisées seront : l'entretien individuel lors des étapes de relation et de réflexion individuelle; l'entretien de groupe lors des étapes de clarification, de négociation, de discussion et de bilan; l'observation directe lors de l'étape d'action; le journal de bord des participants et du chercheur tout au long du processus de recherche.

\section{Conclusion}

Ce projet de recherche aura des retombées sociales et scientifiques significatives. Au niveau social, des stratégies d'évaluation seront développées, opérationnalisées et améliorées pour ensuite être partagées à la communauté des enseignants d'ÉPS afin de leur offrir des pistes de réflexion pour optimiser leurs pratiques évaluatives en ÉS. Au niveau scientifique, cette recherche contribuera à améliorer les connaissances actuelles sur la mise en place d'un dispositif d'accompagnement professionnel visant l'autonomisation des personnes accompagnées. Elle permettra aussi le développement d'un modèle intégrateur afin d'opérationnaliser une telle démarche. 


\section{Références}

Alfrey, L., Webb, L. et Cale, L. (2012). Physical education teachers' continuing professional development in health-related exercise: A figurational analysis. European Physical Education Review, 18(3), 361-379. http://dx.doi.org/10.1177/1356336x12450797

Biémar, S. (2012). Accompagner un groupe d'enseignants dans une école : une grille de compétences. Dans É. Charlier et S. Biémar (dir.), Accompagner : un agir professionnel (p. 17-33). Bruxelles : De Boeck. http://dx.doi.org/10.3917/dbu.charl.2011.01.0017

Grenier, J. (2009). Léducation à la santé dans les programmes scolaires québécois. Formation et profession, 16(2), 15-18. Repéré à http://www.crifpe.ca/download/verify/706

Guay, M.-H. et Prud'Homme, L. (2011). La recherche-action. Dans T. Karsenti et L. Savoie-Zajc (dir.), La recherche en éducation : étapes et approches (3éd., p. 183-211). Québec, QC : Éditions du Renouveau Pédagogique.

Hunzicker, J. (2010). Characteristics of Effective Professional Development: A Checklist. Peoria, IL : Department of Teacher Education, Bradley University.

Lafortune, L., Lepage, C., Persechino, F. et Bélanger, K. (2008). Un modèle d'accompagnement professionnel d'un changement : pour un leadership novateur. Québec, QC : Les Presses de l'Université du Québec.

McNiff, J. et Whitehead, J. (2011). All you need to know about action research (2 éd.). Thousand Oaks, CA : SAGE Publications.

Stringer, E. T. (2007). Action Research (3éd.). Thousand Oaks, CA : SAGE Publications.

Turcotte, S. (2010). Problématisation : l'éducation à la santé et l'éducation physique. Dans J. Grenier, J. Otis et G. Harvey (dir.), Faire équipe pour une éducation à la santé en milieu scolaire (p. 25-48). Québec, QC : Les Presses de l'Université du Québec.

Turcotte, S., Grenier, J., Rivard, M.-C., Beaudoin, C., Roy, M. et Goyette, R. (2011). Besoins de formation continue d'éducateurs physiques et à la santé. Nouveaux cahiers de la recherche en éducation, 14(2), 79-95. http://dx.doi.org/10.7202/1008913ar

\section{Pour citer cet article}

Bezeau, D. (2016). L'accompagnement d'enseignants d'éducation physique et à la santé dans la mise en place de stratégies visant à optimiser leurs pratiques évaluatives en éducation à la santé. Formation et profession, 24(1), 70-73. http://dx.doi.org/10.18162/fp.2016.a87 